\title{
DIFFRACTION TOMOGRAPHY FROM SINGLE-MOLECULE LOCALIZATION MICROSCOPY: NUMERICAL FEASIBILITY
}

\author{
Thanh-an Pham ${ }^{\star} \quad$ Emmanuel Soubies ${ }^{\dagger} \quad$ Ferréol Soulez $^{\ddagger} \quad$ Michael Unser $^{\star}$ \\ * Biomedical Imaging Group, École polytechnique fédérale de Lausanne, \\ Lausanne, Switzerland. \\ † IRIT, Université de Toulouse, CNRS, Toulouse, France. \\ ‡ Univ Lyon, Univ Lyon1, Ens de Lyon, CNRS, Centre de Recherche Astrophysique \\ de Lyon UMR5574, F-69230, Saint-Genis-Laval, France.
}

\begin{abstract}
Single-molecule localization microscopy (SMLM) is a fluorescence microscopy technique that achieves super-resolution imaging by sequentially activating and localizing random sparse subsets of fluorophores. Each activated fluorophore emits light that then scatters through the sample, thus acting as a source of illumination from inside the sample. Hence, the sequence of SMLM frames carries information on the distribution of the refractive index of the sample. In this proof-of-concept work, we explore the possibility of exploiting this information to recover the refractive index of the imaged sample, given the localized molecules. Our results with simulated data suggest that it is possible to exploit the phase information that underlies the SMLM data.
\end{abstract}

Index Terms - Fluorescence microscopy, super-resolution, refractive index recovery

\section{INTRODUCTION}

Single-molecule localization microscopy (SMLM) is a powerful tool to visualize biological structures below the diffraction limit. In SMLM, structures within the sample are labeled with fluorophores that emit fluorescence. The contribution of each activated fluorophore (point source) is a point-spread function (PSF)-like image. By randomly activating a subset of them, SMLM temporally separates these PSFs that would be otherwise overlapping. Then, dedicated algorithms [1] are deployed to localize each activated fluorophore with a precision of the order of $10-20 \mathrm{~nm}$ in the best-case scenario [2-4]. The output of these algorithms is a list of positions of the fluorescent emitters; this list can be used to render a super resolved image. Three-dimensional imaging is also possible by engineering the PSF so that it encodes the axial position [5] or by acquiring simultaneous focal planes [6].

The present work is motivated by the following observations of Gilbert et al. [7]: an emitter produces a point-source illumination that scatters through the sample before being captured by the camera (Figure 1). In other words, SMLM acquisitions can be seen as the measurements of an optical-diffraction tomography system with point-source illuminations. This observation makes it possible to recover the distribution of the refractive index (RI) by solving an

T-A.P. and M.U. were supported by the European Research Council (ERC) under the European Union's Horizon 2020 research and innovation programme (Grant Agreement No. 692726 GlobalBioIm). E.S. was partly supported by the GDR ISIS (project FiMOSuReMi) and the LabEx CIMI (ANR-11-LABX-0040)

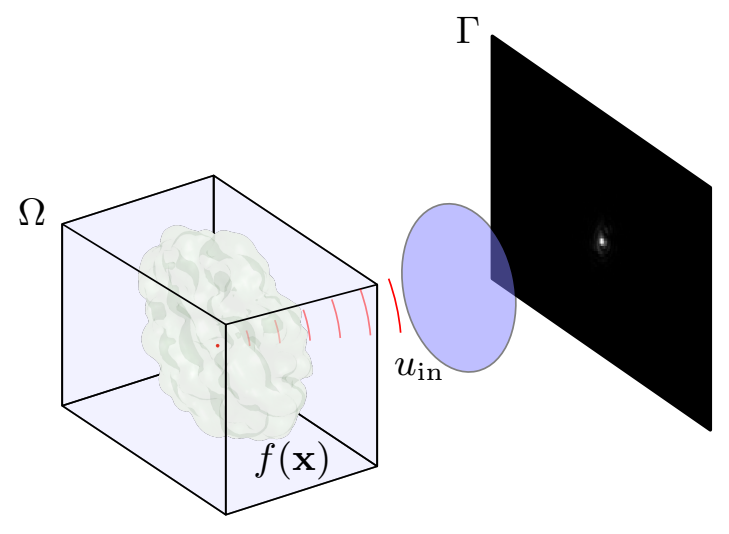

Fig. 1: Single-molecule localization microscopy. A fluorophore emits fluorescent light which scatters through the sample. The intensity of the total field is focused by a lens on a camera sensor.

inverse-scattering problem. This interpretation of the acquisition setup would ideally complete the original purpose of SMLM. Indeed, it offers a unique combination of structural (RI) and functional (fluorescence) information about the sample from a single acquisition of a sequence of SMLM frames, similar to the recent computational hybrid imaging approach proposed in [8]. Moreover, the reconstructed RI can be exploited to improve the localization of the fluorescent molecules in SMLM [9]. However, Gilbert et al. [7] assumed access to complex-valued SMLM measurements (including the phase). In real life, SMLM measurements are phaseless and only contain the intensity information, which makes the inversescattering problem more difficult to solve. Furthermore, they modeled the wave propagation through the sample via a weak-scattering approximation [10]. This potentially leads to underestimated refractive indices [11].

In this proof-of-concept work, we investigate the recovery of refractive indices from intensity-only measurements. To do so, we rely on a more accurate nonlinear model of the propagation of the light [12].

\section{PHYSICAL MODEL}

In SMLM, the fluorophores are activated randomly. For the sake of clarity, we assume that there is only one activated fluorophore 

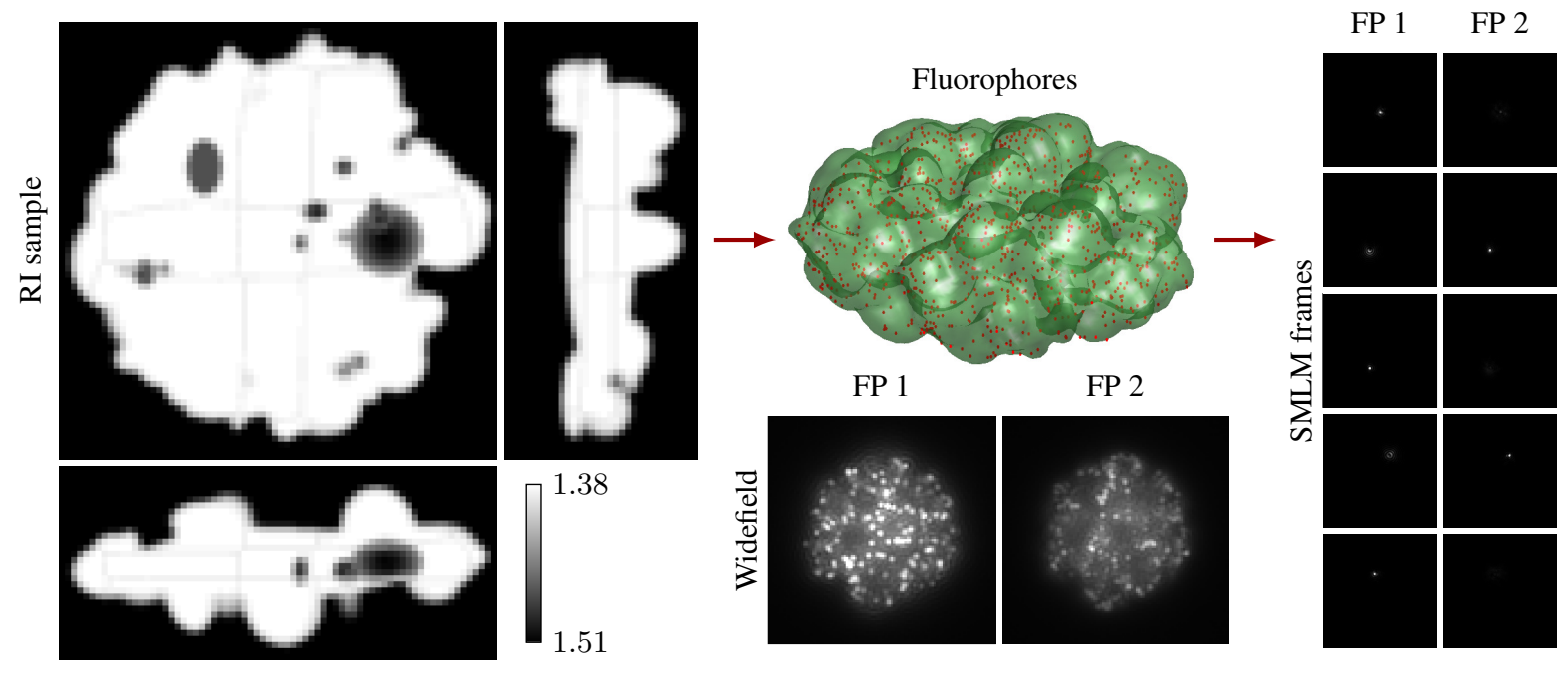

Fig. 2: Simulation setup. The RI volume immersed in oil $\left(n_{b}=1.51\right)$ is populated with fluorophores which sequentially emit a spherical wave. The wave is propagated through the sample using the Lippmann-Schwinger model. Two focal planes (with pupil functions) are acquired. The widefield images are generated by summing up all SMLM frames.

per frame. We describe our physical model within the scalar diffraction theory. Let us consider a sample of refractive index (RI) $\eta: \Omega \rightarrow \mathbb{R}$ with $\Omega \subset \mathbb{R}^{3}$. When activated, the $l$ th fluorophore at the three-dimensional position $\mathbf{x}_{l} \in \Omega$ emits a spherical wave of intensity $a_{l}>0$

$$
u_{\text {in }}\left(\mathbf{x}, \mathbf{x}_{l}, a_{l}\right)=a_{l} \frac{\exp \left(\mathrm{j} k_{\mathrm{b}}\left\|\mathbf{x}-\mathbf{x}_{l}\right\|_{2}\right)}{4 \pi\left\|\mathbf{x}-\mathbf{x}_{l}\right\|_{2}},
$$

where $\mathrm{j}$ is the imaginary unit and $k_{\mathrm{b}}=\frac{2 \pi \eta_{\mathrm{b}}}{\lambda}$ is the wavenumber determined by the wavelength $\lambda$ and the RI $\eta_{\mathrm{b}}>1$ of the medium. The spherical wave acts as an "incident" field that illuminates from within the sample. The wave then scatters through the sample, which produces a total field $u_{l}: \mathbb{R}^{3} \rightarrow \mathbb{C}$. The scattering is governed by the Lippmann-Schwinger equation

$$
u_{l}(\mathbf{x})=u_{\text {in }}\left(\mathbf{x}, \mathbf{x}_{l}, a_{l}\right)+\int_{\Omega} g(\mathbf{x}-\mathbf{z}) f(\mathbf{z}) u_{l}(\mathbf{z}) \mathrm{d} \mathbf{z}
$$

where $f(\mathbf{x})=k_{\mathrm{b}}^{2}\left(\frac{\eta(\mathbf{x})^{2}}{\eta_{\mathrm{b}}^{2}}-1\right)$ is the scattering potential and $g: \mathbb{R}^{3} \rightarrow \mathbb{C}^{3}$ is the Green function that corresponds to the centered spherical wave $u_{\text {in }}(\mathbf{x}, \mathbf{0}, 1)$ [13]. Then, the total field goes through an optical system which, in the Fourier domain, is modeled as the pointwise multiplication with a pupil function $P: \mathbb{R}^{2} \rightarrow \mathbb{C}$. (This also includes the effect of optical refocusing.) Finally, the intensity of the resulting wave is recorded by a camera on a plane $\Gamma$.

Let us discretize $\Omega$ into $N=n^{3}$ voxels. The discrete nonlinear forward model $\mathbf{H}_{l}: \mathbb{R}^{N} \rightarrow \mathbb{R}^{M}$ is then defined by $[11,12,14]$

$$
\begin{aligned}
\mathbf{H}_{l} & : \mathbf{f} \mapsto\left|\mathbf{P}\left(\tilde{\mathbf{G}} \operatorname{diag}(\mathbf{f}) \mathbf{u}_{l}+\mathbf{u}_{\mathrm{in}, l}^{\Gamma}\right)\right|^{2} \\
& \text { with } \mathbf{u}_{l}=(\mathbf{I}-\mathbf{G} \operatorname{diag}(\mathbf{f}))^{-1} \mathbf{u}_{\mathrm{in}, l},
\end{aligned}
$$

where $\mathbf{f} \in \mathbb{R}^{N}, \mathbf{u}_{\text {in }, l} \in \mathbb{C}^{N}$, and $\mathbf{u}_{l} \in \mathbb{C}^{N}$ are sampled version of $f$, $u_{\text {in }}\left(\cdot, \mathbf{x}_{l}, a_{l}\right)$, and $u_{l}$ within $\Omega$, respectively. The matrix $\mathbf{I} \in \mathbb{R}^{N \times N}$ is an identity, while $\operatorname{diag}(\mathbf{f}) \in \mathbb{R}^{N \times N}$ is a diagonal matrix formed out of the entries of $\mathbf{f}$. The matrix $\mathbf{G} \in \mathbb{C}^{N \times N}$ encodes the convolution with the Green function in (2). Similarly, $\tilde{\mathbf{G}} \in \mathbb{C}^{M^{\prime} \times N}$ is a matrix that, given $\operatorname{diag}(\mathbf{f}) \mathbf{u}_{l}$, gives the scattered field at the measurement plane(s) $\Gamma$. The vector $\mathbf{u}_{\mathrm{in}, l}^{\Gamma} \in \mathbb{C}^{M^{\prime}}$ is the incident field at the measurement plane(s) $\Gamma$. Finally, $\mathbf{P} \in \mathbb{C}^{M \times M^{\prime}}$ models the effect of the pupil function $P$ and $|\cdot|^{2}$ denotes the pointwise-squared magnitude. The discretization of the Green function and the spherical wave is thoroughly described in [12].

In this proof-of-concept work, we assume that the positions $\left\{\mathbf{x}_{l} \in \mathbb{R}^{3}\right\}_{l=1}^{L}$ of the $L$ fluorophores are known exactly, as well as the numbers $\left\{a_{l}>0\right\}_{l=1}^{L}$ of emitted photons. In addition, we consider a biplane configuration [6] that requires the simulation of two pupil functions with separate focal planes. To keep the notation simple, we shall use a single matrix $\mathbf{P} \in \mathbb{C}^{M \times M^{\prime}}$ to represent the effect of the two pupil functions (i.e., two focal planes).

To compare with the best-case scenario, we also consider complex measurements. The corresponding forward model $\mathbf{H}_{l}^{\mathrm{c}}: \mathbb{R}^{N} \rightarrow$ $\mathbb{R}^{M}$ is the same as (3) but without the pointwise-squared magnitude. The initial guess was derived from the widefield images (Figure 3 left).

Finally, our noise model is

$$
\begin{aligned}
& \mathbf{y}_{l}^{\mathrm{c}}=\mathbf{H}_{l}^{\mathrm{c}}(\mathbf{f})+\mathbf{n}_{l}^{\mathrm{r}}+\mathrm{j} \mathbf{n}_{l}^{\mathrm{i}} \\
& \mathbf{y}_{l}=\left|\mathbf{y}_{l}^{\mathrm{c}}\right|^{2} \quad \text { for all } l=1, \ldots, L,
\end{aligned}
$$

where $\left\{\mathbf{y}_{l}^{\mathrm{c}} \in \mathbb{C}^{M}\right\}_{l=1}^{L},\left\{\mathbf{y}_{l} \in \mathbb{R}^{M}\right\}_{l=1}^{L}$ are the complex and intensity measurements, respectively. The vectors $\mathbf{n}^{\text {r,i }} \in \mathbb{R}^{M}$ represent Gaussian noise in the real and imaginary parts, respectively. In this work, we simulated a $1 \mathrm{~dB}$ noise with respect to $\mathbf{H}_{l}^{\mathrm{c}}(\mathbf{f})$. We added the noise in the complex domain in the interest of fairness when comparing the complex and intensity-only settings.

\section{PROBLEM FORMULATION}

To reconstruct the scattering potential from $\left\{\mathbf{y}_{l} \in \mathbb{R}^{M}\right\}_{l=1}^{L}$, we adopt the variational formulation

$$
\hat{\mathbf{f}} \in\left\{\arg \min _{\mathbf{f} \in \mathbb{R}_{\geq 0}^{N}}\left(\sum_{l=1}^{L}\left\|\mathbf{H}_{l}(\mathbf{f})-\mathbf{y}_{l}\right\|_{2}^{2}+\tau \operatorname{TV}(\mathbf{f})\right)\right\},
$$




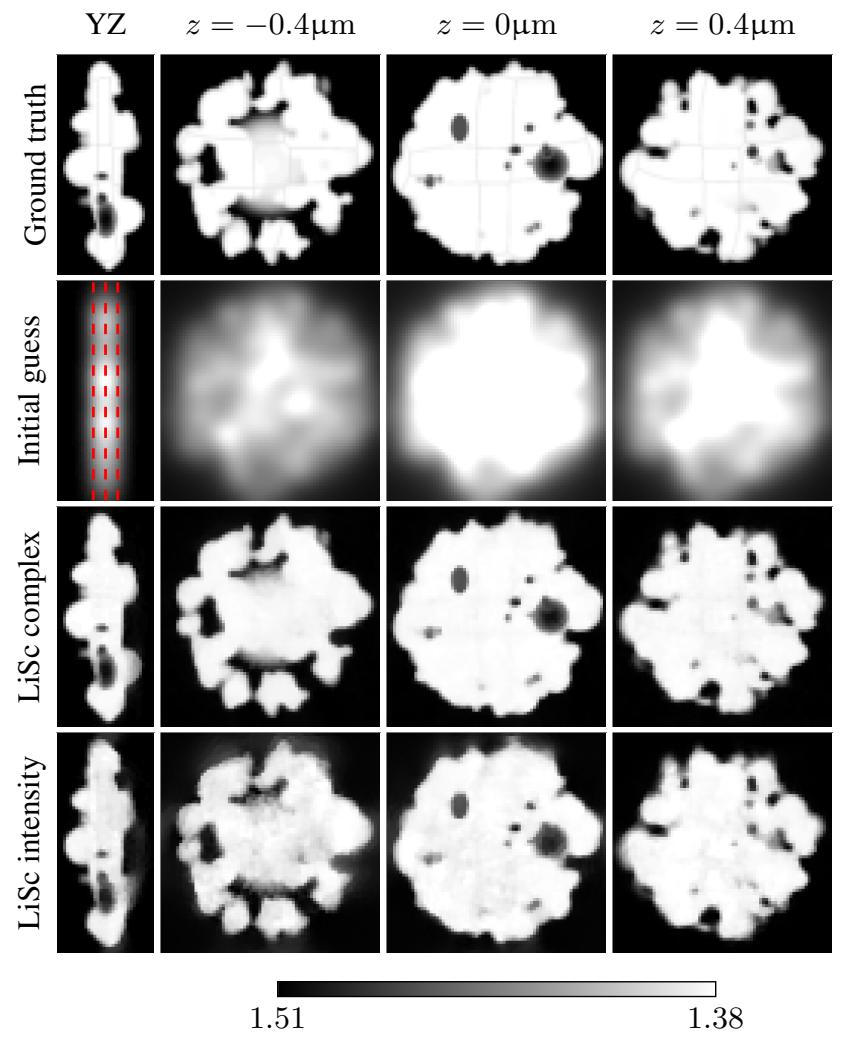

Fig. 3: Reconstructions of the RI volume. LiSc complex: LippmannSchwinger model from complex measurements. LiSc intensity: Lippmann-Schwinger model from intensity measurements.

where TV is the total variation seminorm (TV) [15] and $\tau>0$ is a trade-off parameter. We solve (5) with a stochastic version of the accelerated forward-backward splitting algorithm [16]. The gradient of the data-fidelity term is computed as described in [11]. To compute the proximity operator of $\mathrm{TV}$, we adopt the fast gradient projection [17]. The whole algorithm is implemented within the framework of the GlobalBioIm library [18]. The parameter $\tau$ was tuned optimally by performing a grid search for each reconstruction.

\section{NUMERICAL EXPERIMENTS}

We simulated the acquisition of one-thousand SMLM frames. The sample is a virtual cell immersed in oil $\left(\eta_{\mathrm{b}}=1.51\right)$, fully included in a volume of size $(7.2 \times 7.2 \times 3.6) \mu^{3}{ }^{3}$. The fluorophores were randomly placed on a computer-generated endoplasmic reticulum and cell wall (see gray lines in Figure 2). The emission wavelength is set at $\lambda=647 \mathrm{~nm}$. The two focal planes are placed at $\pm 300 \mathrm{~nm}$ from the center of the sample. The field of view covers $(18.4 \times 18.4) \mu \mathrm{m}^{2}$ with a pixel size of $72 \mathrm{~nm}$. The volume is discretized with a voxel size of $72 \mathrm{~nm}$ as well. In Figure 2, we provide the sample along with a few synthesized SMLM frames.

We reconstruct the volume with high fidelity from the complex measurements as shown in Figure 3. Although less accurate, the reconstruction from intensity-only measurements still captures most of the structures. Only the smallest features are washed out. The objective measures of error are consistent with the visual comparison between both reconstructions (Figure 3). For both setups, we

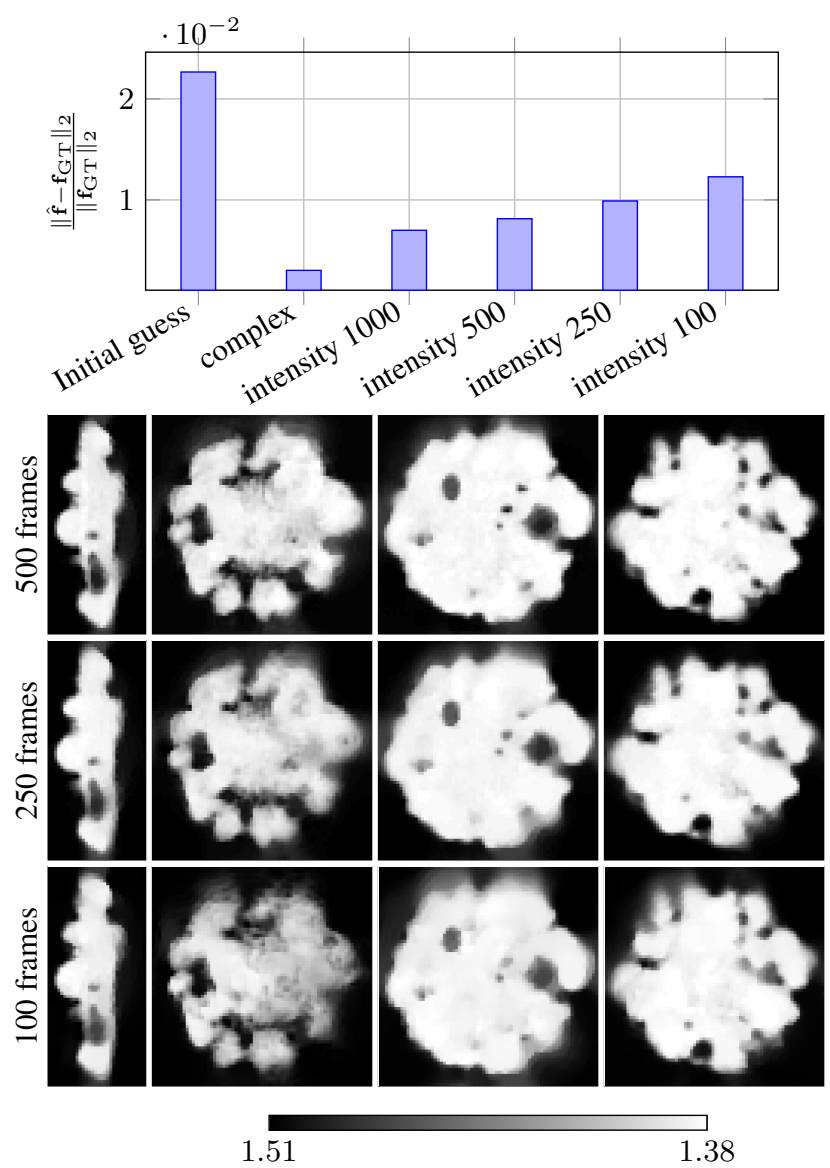

Fig. 4: Top: relative error for different settings. From left to right: initial guess, Lippmann-Schwinger model from complex measurements and 1000 frames, Lippmann-Schwinger model from intensity measurements and $1000,500,250$, and 100 frames, respectively. Bottom: reconstructions of the RI volume with LippmannSchwinger model from intensity measurements and 500, 250, and 100 frames. The axial positions are provided in Figure 3.

can observe that a part of the sample (negative axial position) is reconstructed with lower fidelity (right part of the YZ view). This is because the emitted light is acquired from one side only (the positive axial position). Hence, the frames recorded during the activation of a fluorophore mainly contain information about the structures lying between the fluorophore and the camera, with structures away from the camera being probed less often.

In a second experiment, we assess how the quality of reconstruction varies with respect to the number of frames. We only reconstructed from the intensity measurements and computed the relative error for each case. As shown in Figure 4, the quality of reconstruction remains fairly robust, even when only 100 frames are used.

\section{CONCLUSION}

In this work, we evaluated the possibility to recover the distribution of refractive index (RI) from measurements of single-molecule localization microscopy (SMLM) in a biplane setting. The imaging modality only captures the intensity of the total field, which makes the inverse-scattering problem particularly challenging. To compare 
with the best-case scenario, we also performed the RI reconstruction from simulated complex measurements. Both methods yield reconstructions of high fidelity. In addition, we assessed the robustness of the intensity-based method to the number of available measurements. Future work will address multiple molecules per frame and explore the possibility to use a simpler forward model to accelerate the reconstruction. Although the physical model does not account for polarization, this work is a first step towards an interesting and non-conventional extension of SMLM.

\section{COMPLIANCE WITH ETHICAL STANDARDS}

This article does not contain any studies involving human participants or animals performed by any of the authors.

\section{ACKNOWLEDGMENTS}

There is no potential conflict of interest.

\section{REFERENCES}

[1] Daniel Sage, Thanh-an Pham, Hazen Babcock, Tomas Lukes, Thomas Pengo, Jerry Chao, Ramraj Velmurugan, Alex Herbert, Anurag Agrawal, Silvia Colabrese, Ann Wheeler, Anna Archetti, Bernd Rieger, Raimund Ober, Guy M. Hagen, JeanBaptiste Sibarita, Jonas Ries, Ricardo Henriques, Michael Unser, and Seamus Holden, "Super-resolution fight club: Assessment of 2D and 3D single-molecule localization microscopy software," Nature Methods, vol. 16, no. 5, pp. 387395, 2019.

[2] Samuel T Hess, Thanu PK Girirajan, and Michael D Mason, "Ultra-high resolution imaging by fluorescence photoactivation localization microscopy," Biophysical Journal, vol. 91, no. 11, pp. 4258-4272, 2006.

[3] Michael J Rust, Mark Bates, and Xiaowei Zhuang, "Stochastic optical reconstruction microscopy (STORM) provides subdiffraction-limit image resolution," Nature Methods, vol. 3, no. 10, pp. 793, 2006.

[4] Eric Betzig, George H Patterson, Rachid Sougrat, O Wolf Lindwasser, Scott Olenych, Juan S Bonifacino, Michael W Davidson, Jennifer Lippincott-Schwartz, and Harald F Hess, "Imaging intracellular fluorescent proteins at nanometer resolution," Science, vol. 313, no. 5793, pp. 1642-1645, 2006.

[5] Bo Huang, Wenqin Wang, Mark Bates, and Xiaowei Zhuang, "Three-dimensional super-resolution imaging by stochastic optical reconstruction microscopy," Science, vol. 319, no. 5864, pp. 810-813, 2008.

[6] Manuel F Juette, Travis J Gould, Mark D Lessard, Michael J Mlodzianoski, Bhupendra S Nagpure, Brian T Bennett, Samuel T Hess, and Joerg Bewersdorf, "Three-dimensional sub-100 nm resolution fluorescence microscopy of thick samples," Nature Methods, vol. 5, no. 6, pp. 527-529, 2008.

[7] Anna C Gilbert, Howard W Levinson, and John C Schotland, "Imaging from the inside out: Inverse scattering with photoactivated internal sources," Optics Letters, vol. 43, no. 12, pp. 3005-3008, 2018.

[8] Yi Xue and Laura Waller, "Computational hybrid imaging," arXiv preprint arXiv:2011.11574, 2020.
[9] Fan Xu, Donghan Ma, Kathryn P MacPherson, Sheng Liu, Ye Bu, Yu Wang, Yu Tang, Cheng Bi, Tim Kwok, Alexander A Chubykin, et al., "Three-dimensional nanoscopy of whole cells and tissues with in situ point spread function retrieval," Nature methods, vol. 17, no. 5, pp. 531-540, 2020.

[10] Emil Wolf, "Three-dimensional structure determination of semi-transparent objects from holographic data," Optics Communications, vol. 1, no. 4, pp. 153-156, 1969.

[11] Emmanuel Soubies, Thanh-an Pham, and Michael Unser, "Efficient inversion of multiple-scattering model for optical diffraction tomography," Optics Express, vol. 25, no. 18, pp. 21786-21800, 2017.

[12] Thanh an Pham, Emmanuel Soubies, Ahmed Ayoub, Joowon Lim, Demetri Psaltis, and Michael Unser, "Three-dimensional optical diffraction tomography with Lippmann-Schwinger model," IEEE Transactions on Computational Imaging, vol. 6, pp. 727-738, 2020.

[13] Anda Cornea and P Michael Conn, Fluorescence Microscopy: Super-Resolution and Other Novel Techniques, Elsevier, 2014.

[14] Hsiou-Yuan Liu, Dehong Liu, Hassan Mansour, Petros T Boufounos, Laura Waller, and Ulugbek S Kamilov, "Seagle: Sparsity-driven image reconstruction under multiple scattering," IEEE Transactions on Computational Imaging, vol. 4, no. 1, pp. 73-86, 2017.

[15] Leonid I Rudin, Stanley Osher, and Emad Fatemi, "Nonlinear total variation based noise removal algorithms," Physica D: Nonlinear Phenomena, vol. 60, no. 1-4, pp. 259-268, 1992.

[16] Amir Beck and Marc Teboulle, "A fast iterative shrinkagethresholding algorithm for linear inverse problems," SIAM Journal on Imaging Sciences, vol. 2, no. 1, pp. 183-202, 2009.

[17] Amir Beck and Marc Teboulle, "Fast gradient-based algorithms for constrained total variation image denoising and deblurring problems," IEEE Transactions on Image Processing, vol. 18, no. 11, pp. 2419-2434, 2009.

[18] Emmanuel Soubies, Ferréol Soulez, Michael T. McCann, Thanh-an. Pham, Laurène Donati, Thomas Debarre, Daniel Sage, and Michael Unser, "Pocket guide to solve inverse problems with GlobalBioIm," Inverse Problems, vol. 35, no. 10, pp. 1-20, 2019. 